\title{
Evaluación de la sostenibilidad del soterramiento de la carretera M-30 y la creación de Madrid Río en Madrid, España
}

\author{
A. P. Pérez-Fortes ${ }^{1}$, L. López-de Abajo ${ }^{1}$, M.G. Alberti ${ }^{1}$, J. C. Gálvez ${ }^{1 *}$ \\ *Autor de Contacto: jaime.galvez@upm.es \\ ${ }^{1}$ Departamento de Ingeniería Civil, Construcción, Escuela de Ingenieros de Caminos, Canales y Puertos, \\ Universidad Politécnica de Madrid, Madrid, España
}

\begin{abstract}
RESUMEN
Durante los años 2004 a 2007, se produjo en el área metropolitana de Madrid (España) el soterramiento de parte de la carretera de circunvalación, M-30, y la creación de Madrid Río. Madrid Río se diseñó como un parque lineal situado a ambos lados del río Manzanares que supuso, no sólo un cambio paisajístico y la recuperación del ecosistema fluvial en la zona, sino un punto de encuentro entre los habitantes de cada margen del río con diferentes atracciones de ocio y culturales. De este modo, este artículo pretende realizar un análisis de sostenibilidad del soterramiento de la M-30 y la creación de Madrid Río, 14 años después de su construcción, incluyendo el impacto socioeconómico y ambiental de dichas obras en la zona. Para determinar dicho impacto, se han analizado las condiciones de la zona antes y después de las obras realizadas. Igualmente, se proponen mejoras al proyecto desarrollado considerando la aplicación de materiales de construcción sostenibles y de altas prestaciones. Finalmente, el modelo de análisis propuesto es un modelo multicriterio denominado MIVES (Modelo Integrado para una Evaluación Sostenible), del que se ha concluido que, en función de los indicadores de sostenibilidad elegidos, las obras realizadas en la M-30 y la creación de Madrid Río han influido positiva y significativamente la sostenibilidad de los márgenes del río Manzanares. Sin embargo, el uso de materiales mejorados frente al uso de materiales convencionales no influye significativamente en la sostenibilidad de la obra.
\end{abstract}

Palabras clave: Sostenibilidad; Madrid Río; M-30; Método MIVES; materiales de construcción.

\section{INTRODUCCIÓN}

La ciudad de Madrid, con 3,5 millones de habitantes en su área metropolitana, es la ciudad más grande de España y la cuarta de Europa (Perez-Prada y Monzon 2014), por lo que es una de las 
ciudades con mayor estrés urbano del país. Por estos motivos, en las últimas décadas, la ciudad ha desarrollado una serie de proyectos con el fin de mejorar su sostenibilidad, siendo la red de transportes e infraestructuras una de las áreas más beneficiadas. Es bien sabido que la mejora de la red de transportes de una ciudad mejora la movilidad de sus habitantes y su seguridad, favoreciendo el desarrollo socioeconómico de la misma (Tatari y Kurmapu 2011; Aguado de Cea et al. 2017; Janssen et al. 2019).

En concreto, la ciudad de Madrid conecta con las poblaciones colindantes mediante tres carreteras principales que conforman una serie de anillos concéntricos (M-30, M-40 y M-50). De las tres, la M-30 (Figura 1) es la carretera de circunvalación más próxima al centro urbano, aportando el 24,2 $\%$ del tráfico en el área metropolitana (Perez-Prada y Monzon 2017). Esta carretera, construida en la década de los 70 y remodelada en los años 90, presentaba un diseño heterogéneo donde alternaban tramos urbanos regulados por semáforos con otros de autovía, así como una cantidad excesiva de entradas y salidas del eje principal, generando numerosos accidentes (Monzón de Cáceres et al. 2005). Además, especialmente en el sector suroeste, la M-30 atravesaba superficialmente las áreas urbanas del centro de Madrid, provocando diversos problemas medioambientales como ruidos y contaminación atmosférica (Tatari y Kurmapu 2011). Además, en este tramo, la M-30 ejercía una barrera en la movilidad de los habitantes de ambos márgenes del río Manzanares (Perez-Prada y Monzon 2017).

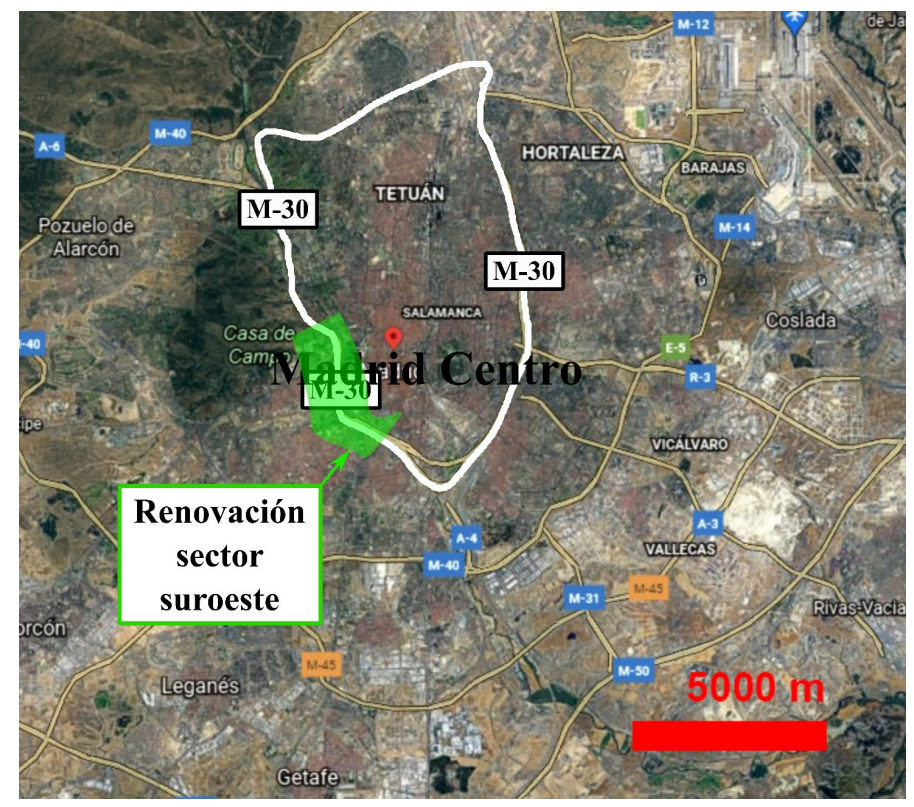

Figura 1. Localización de las obras de rehabilitación de la M-30 y creación de Madrid Río en Madrid (España).

Por estos motivos, el sector suroeste de la M-30 (Figura 1) fue remodelado entre los años 2004 y 2007, con la idea de mejorar la red vial de la carretera, así como el confort y condiciones ambientes de las áreas colindantes. Para ello, se construyeron $8,8 \mathrm{~km}$ de túneles, reubicando y homogeneizando el tráfico de la zona (Perez-Prada y Monzon 2017), lo que redujo la accidentalidad de la zona. Dicho soterramiento de la M-30 hizo también posible la creación de un nuevo corredor verde, Madrid Río, cuya finalidad era reducir el impacto medio ambiental y el efecto barrera provocado por el intenso tráfico de la zona (Monzón de Cáceres et al. 2005; PerezPrada y Monzon 2017; Consejería de Economía y Hacienda de la Comunidad de Madrid 2019). En este trabajose evalúa la sostenibilidad de las obras realizadas en el sector suroeste de la carretera M-30 y de la creación de Madrid Río. El método empleado es el MIVES, Modelo Integrado para 
una Evaluación Sostenible, que es un método de toma de decisiones de tipo multicriterio, capaz de considerar criterios de carácter cualitativo con evaluación cuantitativa, facilitando la integración de ciertos factores ambientales y, especialmente, sociales. Dichos factores han sido seleccionados mediante consenso por los autores de este artículo, considerando tres escenarios: el sector suroeste de la M-30 previo a la construcción de los túneles y la creación de Madrid Río; el mismo sector transcurridos 14 años del enterramiento de la M-30 y de la creación de Madrid Río; el mismo sector considerando el estado actual de la construcción si se hubieran empleado materiales sostenibles y de altas prestaciones tales como áridos reciclados o el uso de hormigones especiales.

\section{PROCEDIMIENTO}

A la hora de enfrentarse al análisis de sostenibilidad de un área, donde no sólo se deben tener en cuenta los factores medioambientales, sino que se debe alcanzar un cierto balance entre dichos factores y otros factores económicos y sociales, los métodos de toma de decisiones multicriterio son, probablemente, los más adecuados (Cafiso et al. 2002; Pujadas et al. 2017; Stojčić et al. 2019). En este contexto, MIVES es un método multicriterio que combina un árbol discriminatorio holístico de requisitos, criterios e indicadores (Pons y Wadel 2011; Aguado de Cea et al. 2016; Aguado de Cea et al. 2017); el concepto de función de valor (Alarcon et al. 2011) y seminarios impartidos por expertos para evaluar las funciones y pesos utilizados en el proceso de jerarquía analítica (Pons y Wadel 2011; Pujadas et al. 2017; Saaty 2012). Este método permite a los investigadores obtener una justificación científica y basada en la sostenibilidad para tomar una decisión entre diferentes alternativas (Pujadas et al. 2017) y obtener un valor o índice de sostenibilidad del problema adimensional, SI (Viñolas Prat et al. 2009).

El árbol de criterio de MIVES se compone de tres niveles: requisitos, criterios e indicadores, que son evaluados mediante funciones de valor, convirtiendo los factores cualitativos en cuantitativos, siendo los expertos quienes ponderan cada requisito, criterio e indicador para evaluar su importancia en el modelo y el SI final (Alberti et al. 2018). De este modo, para el análisis de sostenibilidad propuesto, se tuvieron en cuenta requisitos económicos, medioambientales y sociales de la obra, los criterios o estadios y procesos de la obra que tuvieron un impacto en la zona y los indicadores de dicho impacto durante los diferentes estadios de la obra. Igualmente, tanto a los requisitos, como criterios e indicadores se les confirieron diferentes pesos en el modelo, en función de su importancia relativa en el impacto final. Además, con el fin de evaluar los beneficios ambientales y socioeconómicos de rehabilitación de la zona, se definieron tres alternativas:

a) M-30 no enterrada: en esta situación se consideró el antiguo trazado de la M-30, antes de la construcción de los túneles y creación del parque Madrid Río.

b) Soterramiento de la M-30 mediante el uso de materiales convencionales: es el estado actual de la vía.

c) Soterramiento de la M-30 mediante el uso de materiales mejorados: esta alternativa considera la aplicación de procedimientos y materiales de construcción sostenibles y de altas prestaciones para evaluar su influencia en la sostenibilidad del proyecto.

\section{APLICACIÓN DEL MÉTODO MIVES A LAS OBRAS DE SOTERRAMIENTO DE LA M-30 Y LA CREACIÓN DE MADRID RÍO}

Los túneles de la M-30 se construyeron como un túnel a cielo abierto con muros pantalla y falso techo (junto al río Manzanares) y un túnel construido mediante tuneladora (Martínez Paricio 2013) en otra de las zonas. Dichos túneles cuentan con altas medidas de seguridad para la prevención de 
accidentes e incendios, así como varias unidades y dispositivos de control para garantizar una calidad del aire adecuada. De hecho, están equipados con diversas áreas de ventilación de unos 600 $\mathrm{m}$ de longitud para diluir la concentración de contaminantes en el interior (del Rey Llorente et al. 2009).

Igualmente, Madrid Río es un parque lineal con 429 hectáreas de nuevas áreas verdes distribuidas a lo largo de la ribera del río Manzanares con el objetivo de integrar el entorno fluvial en el paisaje urbano de Madrid (Hernández-Lamas et al. 2016). Así, el parque ofrece no solo una mejora de las condiciones ambientales si no también la eliminación de la barrera vial anterior, que separaba el sur del centro de Madrid, produciendo así extensa regeneración urbana de la zona (Bosch Reig et al. 2018).

Teniendo en cuenta el contexto y la finalidad de la obra, se establecieron y ponderaron los requisitos económicos (Tabla 1), medioambientales (Tabla 2) y sociales (Tabla 3) de la obra realizada, así como los diferentes criterios e indicadores, teniendo en cuenta las siguientes consideraciones:

1) Requisitos económicos (Tabla 1): Es bien conocido que las carreteras promueven la economía y el desarrollo, cambiando los patrones de movilidad de la población y mejorando la accesibilidad de una zona, lo que repercute directamente en el nivel de vida de las personas que residen en zonas urbanas (Junyent 2001; Aguado de Cea et al. 2016). Por tanto, es relevante evaluar el equilibrio entre los costes directos de la construcción y los beneficios económicos indirectos generados por la renovación de las áreas circundantes.

Tabla 1. Criterios e indicadores económicos considerados en el análisis MIVES

\begin{tabular}{|c|c|c|}
\hline $\begin{array}{l}\text { Requerimiento } \\
\text { (\% peso) }\end{array}$ & Criterios (\% peso) & Indicadores (\% Peso) \\
\hline \multirow{8}{*}{$\begin{array}{l}\text { R1. Económico } \\
40 \%\end{array}$} & $\begin{array}{c}\text { C1 Costes totales. Directos } \\
+ \text { Indirectos } 30 \%\end{array}$ & $\begin{array}{l}\text { I1 Costes totales, incluyendo tiempo de } \\
\text { construcción } 100 \%\end{array}$ \\
\hline & C2 Calidad $10 \%$ & I2 Costes de calidad disconforme $100 \%$ \\
\hline & $\begin{array}{l}\text { C3 Desmantelamiento } \\
10 \%\end{array}$ & I3 Costes de desmantelamiento $100 \%$ \\
\hline & \multirow{2}{*}{ C4 Vida útil 30\% } & $\begin{array}{l}\text { I4 Costes de servicio, mantenimiento, energía y } \\
\text { carga de uso } 80 \%\end{array}$ \\
\hline & & $\begin{array}{l}\text { I5 Resiliencia, riesgo de desastre, costes de } \\
\text { reconstrucción y de falta de uso } 20 \%\end{array}$ \\
\hline & \multirow{3}{*}{$\begin{array}{l}\text { C5 Beneficios en la zona } \\
\qquad 20 \%\end{array}$} & I6 Mercado inmobiliario $50 \%$ \\
\hline & & I7 Incremento del turismo $15 \%$ \\
\hline & & I8 Impulso cultural $35 \%$ \\
\hline
\end{tabular}

2) Requisitos ambientales: Los criterios e indicadores estudiados están relacionados con el material y consumo de energía durante la construcción, explotación y mantenimiento de la infraestructura. Igualmente, el transporte por carretera es responsable del 93\% del impacto medioambiental generado en Europa (van Essen et al. 2011; Sánchez et al. 2018). Por todo ello, las emisiones contaminantes y acústicas durante la construcción, explotación y mantenimiento de la carretera y túneles analizados se han considerado también en el análisis (Tabla 2). Por último, dado que uno de los principales objetivos de la renovación del la M30 supuso la vinculación del sistema fluvial del río Manzanares con el paisaje urbano de Madrid (Hernández-Lamas et al. 2016; Perini y Sabbion 2016), la recuperación de los ecosistemas fluviales del Manzanares en el tramo madrileño del río y criterios relacionados también fueron considerados. 
Tabla 2. Criterios e indicadores medioambientales considerados en el análisis MIVES

\begin{tabular}{|c|c|c|}
\hline $\begin{array}{l}\text { Requerimient } \\
\text { o }(\% \text { peso) }\end{array}$ & Criterios (\% peso) & Indicadores (\% Peso) \\
\hline \multirow{26}{*}{$\begin{array}{l}\mathrm{R} 2 . \\
\text { Medioambient } \\
\text { al } 30 \%\end{array}$} & \multirow{6}{*}{$\begin{array}{l}\text { C6 Consumo de material durante la } \\
\text { etapa de construcción } 15 \%\end{array}$} & I9 Cemento $25 \%$ \\
\hline & & I10 Áridos $10 \%$ \\
\hline & & $\begin{array}{l}\text { I11 Refuerzo de hormigón (aceros y } \\
\text { fibras) } 15 \%\end{array}$ \\
\hline & & I12 Agua $25 \%$ \\
\hline & & I13 Materiales Auxiliares 15\% \\
\hline & & I14 Material reciclado $10 \%$ \\
\hline & \multirow{6}{*}{$\begin{array}{l}\text { C7 Consumo de material durante la } \\
\text { etapa de mantenimiento } 20 \%\end{array}$} & I9 Cemento $25 \%$ \\
\hline & & I10 Áridos 10\% \\
\hline & & $\begin{array}{l}\text { I11 Refuerzo de hormigón (aceros y } \\
\text { fibras) } 15 \%\end{array}$ \\
\hline & & I12 Agua $25 \%$ \\
\hline & & I13 Materiales auxiliares 15\% \\
\hline & & I14 Materiales reciclados $10 \%$ \\
\hline & \multirow{2}{*}{$\begin{array}{l}\text { C8 Emisiones durante la etapa de } \\
\text { construcción } 10 \%\end{array}$} & I15 Potencial impacto en el clima $80 \%$ \\
\hline & & I16 Residuos totales $20 \%$ \\
\hline & \multirow{2}{*}{$\begin{array}{c}\text { C9 Emisiones durante la etapa de } \\
\text { mantenimiento } 10 \%\end{array}$} & I15 Potencial impacto en el clima $80 \%$ \\
\hline & & I16 Residuos totales $20 \%$ \\
\hline & \multirow{4}{*}{$\begin{array}{c}\text { C10 Emisiones durante la } \\
\text { explotación de la infraestructura } \\
20 \%\end{array}$} & $\begin{array}{l}\text { I17 Almacenamiento de } \mathrm{CO} 2 \text { por } \\
\text { materiales derivados del cemento } 15 \%\end{array}$ \\
\hline & & $\begin{array}{l}\text { I18 Calidad del aire (carretera, túnel y } \\
\text { alrededores) } 30 \%\end{array}$ \\
\hline & & $\begin{array}{l}\text { I19 Emisiones dependientes del límite } \\
\text { de velocidad } 25 \%\end{array}$ \\
\hline & & $\begin{array}{l}\text { I20 Emisión de partículas durante el uso } \\
\text { de la infraestructura } 30 \%\end{array}$ \\
\hline & \multirow{3}{*}{ C11 Energía 10\% } & I21 Energía de la infraestructura 20\% \\
\hline & & I22 Energía de construcción 40\% \\
\hline & & $\begin{array}{l}\text { I23 Energía de servicio y mantenimiento } \\
40 \%\end{array}$ \\
\hline & \multirow{3}{*}{$\begin{array}{l}\text { C12 Ecosistema del río Manzanares } \\
\qquad 15 \%\end{array}$} & I24 Contaminación $50 \%$ \\
\hline & & $\begin{array}{l}\text { I25 Recuperación del sistema fluvial } \\
35 \%\end{array}$ \\
\hline & & $\begin{array}{l}\text { I26 Ecosistema (flora y fauna endémica) } \\
15 \%\end{array}$ \\
\hline
\end{tabular}

3) Requisitos sociales: En este apartado se evalúan los efectos positivos y negativos que la renovación de la M-30 y la creación de Madrid Río implica para los trabajadores, usuarios y vecinos y su forma de vida durante el diseño del proyecto, construcción y uso de la carretera. En este caso, los criterios seleccionados se basaron en la premisa de que las 
carreteras pueden producir directa o físicamente e indirecta o fisiológica y psicológicamente efectos sobre los trabajadores, los usuarios y los residentes (Tabla 3) (Cramphorn y Davies 2004).

Tabla 3. Criterios e indicadores sociales considerados en el análisis MIVES

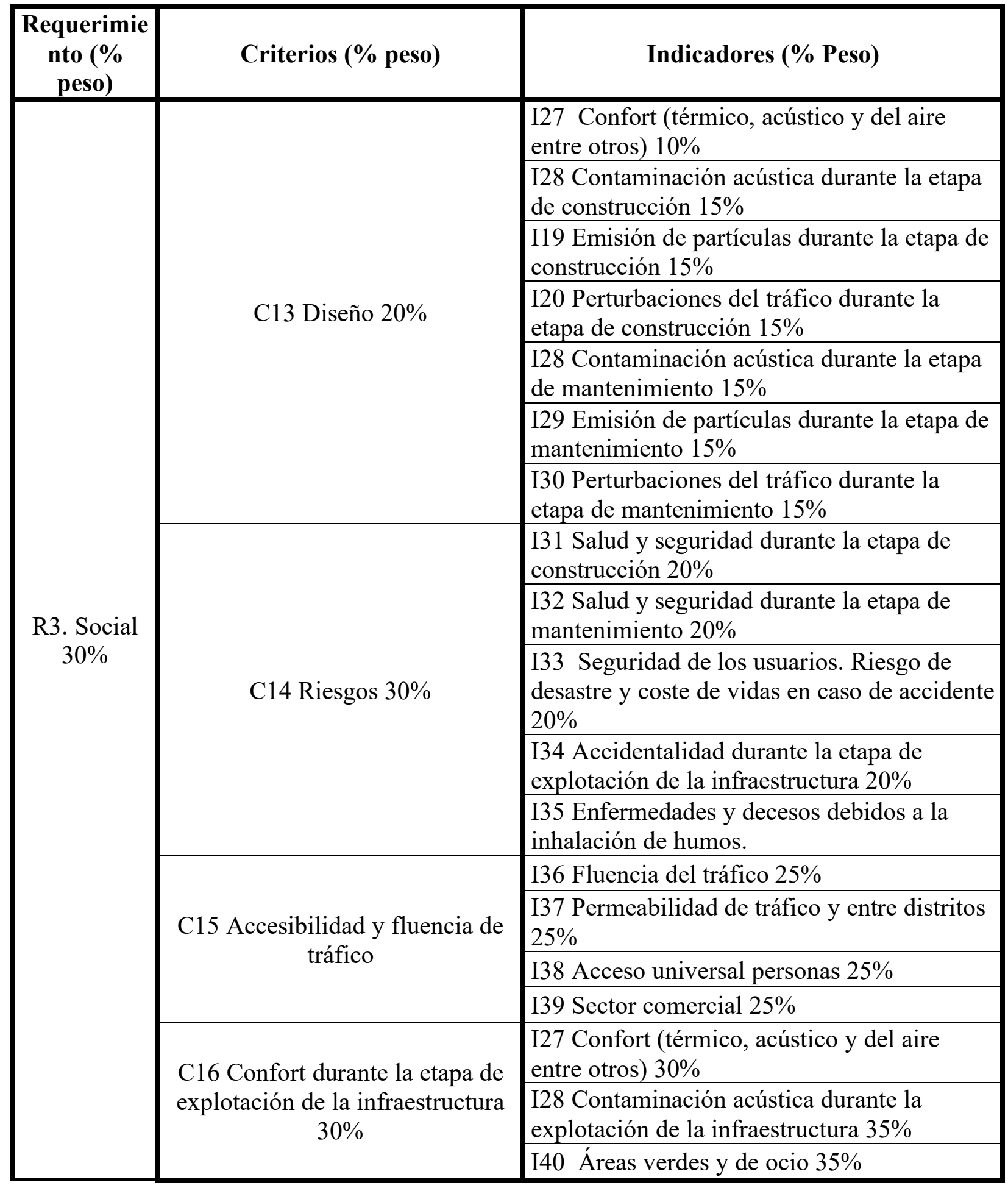

Una vez establecidos los requisitos, criterios e indicadores (Tablas 1, 2 y 3), se aplican funciones de valor para cada indicador, convirtiendo las magnitudes y unidades de los criterios en un valor 
común adimensional (Pons y Wadel 2011). Dicho valor, en este caso, se estableció en un rango entre 0 y 10 , siendo 10 el grado más alto de satisfacción social y sostenibilidad y 0 el más bajo. Por último, se consideró el SI de cada alternativa definida ( $a, b$ y $c$ ) de la M-30: es decir, no soterrado, soterrado empleando materiales convencionales y soterrados aplicando materiales de construcción de prestaciones mejoradas.

\section{RESULTADOS Y DISCUSIÓN DEL ANÁLISIS MIVES}

Los valores de cada requisito considerado para el análisis MIVES de sostenibilidad de la renovación de la M-30, así como el SI para las tres soluciones alternativas estudiadas, se muestran en la Tabla 4.

Se puede observar que el SI de la situación considerando el soterramiento de la M-30 duplica el SI del trazado anterior de la vía. Sin embargo, el SI de la M-30 considerando su soterramiento empleando nuevos materiales es solo 0,5 más alto que usando materiales convencionales. Estos resultados pueden atribuirse a la premisa de que la regeneración de la M-30 se diseñó teniendo en cuenta los aspectos medioambientales y socioeconómicos necesarios para mejorar las condiciones de la zona (Cramphorn y Davies 2004; Bosch Reig et al. 2018). En este sentido, se puede observar que varios de los indicadores seleccionados tienen el mismo valor en cuanto al uso o no de productos más ecológicos y de altas prestaciones (Tabla 4).

Tabla 4. Principales resultados del análisis de sostenibilidad aplicado a las obras de rehabilitación de la M-30 y creación de Madrid Río mediante el método MIVES

\begin{tabular}{|c|c|c|c|}
\hline $\begin{array}{c}\text { Requerimientos } \\
\text { (\% peso) }\end{array}$ & $\begin{array}{c}\text { Alternativa 1. } \\
\text { M-30 no } \\
\text { enterrada }\end{array}$ & $\begin{array}{c}\text { Alternativa 2. } \\
\text { Soterramiento M-30 y } \\
\text { materiales } \\
\text { convencionales }\end{array}$ & $\begin{array}{c}\text { Alternativa 3. } \\
\text { Soterramiento M-30 y uso } \\
\text { de materiales especiales }\end{array}$ \\
\hline $\begin{array}{c}\text { R1. Económicos } \\
40 \%\end{array}$ & 1,50 & 2,29 & 2,32 \\
\hline $\begin{array}{c}\text { R2. } \\
\text { Medioambientales } \\
30 \%\end{array}$ & 1,17 & 1,62 & 1,99 \\
\hline R3. Sociales 30\% & 0,77 & 2,34 & 2,42 \\
\hline $\begin{array}{c}\text { Índice de } \\
\text { Sostenibilidad (SI) }\end{array}$ & $\mathbf{3 , 4 3}$ & $\mathbf{6 , 2 6}$ & $\mathbf{6 , 7 4}$ \\
\hline
\end{tabular}

\subsection{Criterios económicos}

Desde el punto de vista económico, los indicadores basados en los costes de la infraestructura, especialmente su renovación y desmantelamiento, tienen un valor contrario a la renovación de la M-30 (C1, C3, Tablas 1 y 4). Sin embargo, la renovación del trazado implicó una serie de beneficios económicos indirectos del área circundante a tener en cuenta, especialmente a largo plazo (C4, C5, Tablas 1 y 4). Además, hay que tener en cuenta el envejecimiento de la infraestructura y los costes entonces de mantenimiento de la misma. Entonces, si se tiene en cuenta la vida útil de la construcción, la primera alternativa (M-30 no soterrada) será la alternativa más penalizada, dado que las estructuras son más antiguas y requieren, por tanto, más operaciones de mantenimiento. Igualmente, mantener el trazado original de la M-30 hubiera supuesto nuevas remodelaciones e intervenciones para cumplir los estándares españoles actuales en cuento a diseño (Ministerio de Fomento 2014) y, por ejemplo, contaminación acústica en zonas urbanas y residenciales 
(Ministerio de Presidencia 2007; Consejería de Economía y Hacienda de la Comunidad de Madrid 2019). En cuanto a la construcción de los túneles con materiales sostenibles y de altas prestaciones, cabe destacar que generaría un incremento de costes durante la construcción de la obra, pero, a largo plazo, supondría una reducción en los costes de mantenimiento, al ser materiales más duraderos. Finalmente, se ha tenido en cuenta el impacto que ha tenido la creación de Madrid Río en la economía de los barrios afectados por la obra. El precio medio de las viviendas en torno a la M-30, entre 2005 y 2015 aumentó en aproximadamente 500 euros por metro cuadrado debido a las mejoras proporcionadas por las obras (Ortiz de Andrés 2016). Este incremento en el valor de los inmuebles está directamente relacionado con el impulso de las actividades culturales y de ocio, así como las comerciales, en las proximidades de Madrid Río (Tabla 4).

\subsection{Criterios medioambientales}

Desde el punto de vista medioambiental, el soterramiento de la M-30 supuso el consumo de materiales de construcción como es el hormigón, frente a la alternativa de mantener el trazado original de la carretera (I3-I9, Tablas 2 y 4). Por tanto, ha de tenerse en cuenta como indicadores de impacto negativo medioambiental, el consumo de materias primas, tales como cemento, áridos y/o agua. Este impacto, además, aumentaría, por ejemplo, si se emplearan hormigones de alta resistencia, que requieren áridos especiales de alta resistencia y un mayor consumo de cemento (Zhou et al. 1995; Kiliç et al. 2008). Por el contrario, el uso de este tipo de materiales, más duraderos, reduciría las acciones de mantenimiento y renovación en la zona, reduciéndose entonces el impacto ambiental de estas acciones en la zona. Además, el uso de materiales más sostenibles, como es de áridos reciclados, reduciría el impacto de este indicador (I14, Tablas 2 y 4), por lo que el uso de materiales más ecológicos y de altas prestaciones es la alternativa más adecuada de las tres propuestas (C6, Tablas 2 y 4$)$.

En cuanto al nuevo diseño del trazado de la M-30, el enterramiento del tramo de carretera, supuso el aumento de ruidos y emisiones de contaminantes durante la etapa de construcción, pero los beneficios ambientales en cuanto a estos dos factores han sido notables. Sin embargo, el aumento del tráfico, especialmente en el acceso a los túneles aún genera un cierto impacto negativo en la zona, considerándose finalmente el valor de la recuperación como media (I17-I20, Tablas 2 y 4). Finalmente, la creación de Madrid Río ha supuesto la recuperación del sistema fluvial y su ecosistema. El soterramiento de la vía y la reutilización del terreno del entorno como zona verde con más de 33.000 árboles recién plantados 470.000 arbustos, y 210.000 metros cuadrados de pradera de baja demanda de agua, permite una reducción de 35.000 toneladas de $\mathrm{CO}_{2}$ de la contaminación atmosférica, así como la reducción significativa de contaminación acústica en la zona (Bosch Reig et al. 2018). Sin embargo, la mejora de estos indicadores se ha considerado media porque el diseño original de Madrid Río no contribuyó a restaurar las características naturales del río Manzanares y se realizaron acciones adicionales en años posteriores (Da Silva Faustino 2018). Cabe mencionar que los criterios relacionados con la creación del parque, sólo dependen del diseño de la estructura y no del uso de unos u otros materiales (C12, Tablas 2 y 4)

\subsection{Criterios sociales}

Desde el punto de vista social, se han tenido en cuenta las principales perturbaciones (por ejemplo, ruido, emisiones de polvo y otros contaminantes, o la congestión del tráfico), tanto durante las obras, como durante el uso de la infraestructura (Tablas 3 y 4). Por ejemplo, las obras provocaron interrupciones del tráfico y produjo ruido adicional y contaminantes (polvo) en el área. Sin embargo, la comodidad de los usuarios y la población ha incrementado considerablemente con la construcción de los túneles y Madrid Río. El soterramiento de la M-30 y la creación del parque 
Madrid Río también ha redefinido el uso del suelo de la zona, potenciando el ocio y las actividades culturales y deportivas disponibles para residentes y visitantes. También han influido en los patrones de movilidad en el área, aumentando la permeabilidad entre los barrios que habían estado históricamente separados por la barrera del río Manzanares y la M-30 (Da Silva Faustino 2018). Estas mejoras significativas para el uso colectivo del espacio han sido percibidas positivamente por la población, aumentando el confort social en la zona (Tabla 4). Nuevamente, cabe destacar que estos indicadores son directamente dependientes del diseño de la obra, por lo que la aplicación de nuevos materiales tiene nulo o bajo impacto en esta alternativa propuesta.

\section{CONLUSIONES}

A la vista del resultado obtenido del análisis MIVES, aunque se puede concluir que la alternativa existente hoy en día muestra un alto nivel de sostenibilidad, que podría haber sido mejorado utilizando materiales de prestaciones mejoradas y de menor impacto ambiental (como los áridos reciclado). Por lo tanto, los beneficios ambientales y socioeconómicos proporcionados por la renovación del trazado compensan las perturbaciones generales e interrupciones en el tráfico generadas durante las obras.

Aunque el nivel de sostenibilidad obtenido podría haber mejorado ligeramente si se hubieran utilizado nuevos materiales sostenibles y de altas prestaciones, su uso habría implicado una etapa de construcción más prolongada, costes de construcción más altos y, posiblemente la contratación de personal especializado, aumentando así el impacto económico de la obra. Además, el uso de materiales mejorados no afecta a una gran parte de los indicadores medioambientales y socioeconómicos, especialmente durante el diseño y uso de la infraestructura, sólo afectaría a la etapa constructiva y, ligeramente, al uso final de la infraestructura.

Finalmente, MIVES es un método disponible muy adecuado para evaluar la sostenibilidad de las estructuras en diversos escenarios en los que intervienen un elevado número de factores heterogéneos. Esto se debe a que es un métodod multicriterio que tiene capacidad para combinar criterios socioeconómicos y ambientales, así como indicadores cualitativos y cuantitativos.

\section{AGRADECIMIENTOS}

Este trabajo ha sido financiado por el Ministerio de Economía, Industria y Competitividad de España a través del Proyecto Fondo de Investigación PID2019-108978RB-C31. Los autores también quieren agradecer a Calle 30 el apoyo a la Cátedra Universitaria Empresa Calle30-UPM.

\section{REFERENCIAS}

Aguado de Cea, A., Gálvez, J.C. and Fernández-Ordoñez, D., y Albert de la Fuente. 2016. "Sustainability evaluation of the concrete structures". En Second International Conference on Concrete Sustainability (ICCS-16), editado por J.C. Gálvez, A. Aguado, y D. FernándezOrdóñez, 58-71. Madrid (Spain).

Aguado de Cea, A., J. C. Gálvez, A. Aguado Renter, P. Pujadas Álvarez, y D. Fernández-Ordoñez. 2017. "Evaluación de la sostenibilidad de carreteras". Carreteras 4 (213): 8-19. https://upcommons.upc.edu/bitstream/handle/2117/108027/21549563.pdf?sequence=1\&isAl lowed $=\mathrm{y}$.

Alarcon, B., A. Aguado, R. Manga, y A. Josa. 2011. "A value function for assessing sustainability: Application to industrial buildings". Sustainability 3 (1): 35-50. https://doi.org/10.3390/su3010035. 
Alberti, Marcos G., J. C. Gálvez, A. Enfedaque, A. Carmona, C. Valverde, y G. Pardo. 2018. “Use of steel and polyolefin fibres in the La Canda tunnels: Applying MIVES for assessing sustainability evaluation". Sustainability (Switzerland) $10 \quad$ (12): 1-11. https://doi.org/10.3390/su10124765.

Bosch Reig, I., L. Bosch Roig, V. Marcenac, y N. Salvador Luján. 2018. "Linear parks understood as vertebration instruments of the city". En 24th ISUF International Conference. Book of Papers, 983-93. Valencia, Spain: Editorial Universitat Politècnica de València. https://doi.org/10.4995/isuf2017.2017.6136.

Cafiso, S., A. Di Graziano, H. R. Kerali, y J. B. Odoki. 2002. "Multicriteria analysis method for pavement maintenance management". Transportation Research Record, núm. 1816: 73-84. https://doi.org/10.3141/1816-09.

Consejería de Economía y Hacienda de la Comunidad de Madrid. 2019. "Informe Final: Estudio de caso. Impactos del proyecto de soterramiento de Calle 30. Exp. 300/2017/00911”. Madrid (Spain).

https://www.madrid.es/UnidadWeb/Contenidos/EspecialInformativo/AuditoriaDeuda/Infor mesImpacto/ObraSoterramientoM30.pdf.

Cramphorn, B., y R. Davies. 2004. "The social impact of roads: Social capital and road planning". Australian Planner 41 (3): 46-47. https://doi.org/10.1080/07293682.2004.9982372.

Essen, H., A. Schroten, M. Otten, D. Sutter, C. Schreyer, R. Zandonella, M. Maibach, y C. Doll. 2011. "External costs of transport in Europe. Update Study for 2008". A Handbook of Transport Economics. https://doi.org/10.4337/9780857930873.00024.

Hernández-Lamas, P., A. Rubio Gavilán, y J. Bernabeu-Larena. 2016. "Parks and roads build the cities: the M-30 and Madrid-Río project, building landscape". En 11th International Conference Virtual City and Territory: Back to the sense of the city, 415-28. Krakow, Poland: Centre of Land Policy and Valuations (CPSV). Barcelona, Spain. https://upcommons.upc.edu/handle/2117/90986.

Janssen, M. A., J. M. Anderies, A. Baeza, H. L. Breetz, T. Jasinski, H. C. Shin, y S. Vallury. 2019. "Highways as coupled infrastructure systems: an integrated approach to address sustainability challenges". Sustainable and Resilient Infrastructure 00 (00): 1-12. https://doi.org/10.1080/23789689.2019.1708181.

Junyent, R. 2001. "Evaluating the social impact of urban highways. Application to the outer ring road in Barcelona". Luxembourg.

Kiliç, A., C. D. Atiş, A. Teymen, O. Karahan, F. Özcan, C. Bilim, y M. Özdemir. 2008. "The influence of aggregate type on the strength and abrasion resistance of high strength concrete". Cement and Concrete Composites $30 \quad$ (4): 290-96. https://doi.org/10.1016/j.cemconcomp.2007.05.011.

Martínez Paricio, J. I. 2013. "Los túneles de la Calle 30 de Madrid. Características." Safety \& Fire Technique / Bezpieczenstwo i Technika Pozarnicza 30 (2): 127-35.

Ministerio de Fomento. 2014. "Orden Circular 35/2014 Sobre Criterios De Aplicación De Sistemas De Contención De Vehículos”. Madrid (Spain). https://www.fomento.gob.es/NR/rdonlyres/11AFD57B-6604-4E4D-8F1ED4535CB18249/124764/OC352014.pdf.

Ministerio de Presidencia. 2007. Real Decreto 1367/2007, de 19 de octubre, por el que se desarrolla la Ley 37/2003, de 17 de noviembre, del Ruido, en lo referente a zonificación acústica, objetivos de calidad y emisiones acústicas. BOE-A-2007-18397.

Monzón de Cáceres, A., J. M. Pardillo Mayora, L. Vega Báez, J. Bustinduy Fernández, A. de Vicenete González, y M. Pérez Flores. 2005. "El programa de mejoras de la M-30 en el contexto de una estrategia de movilidad sostenible para Madrid." Revista de Obras Públicas 3454: 7-26. http://ropdigital.ciccp.es/pdf/publico/2005/2005_abril_3454_01.pdf. 
Ortiz de Andrés, I. 2016. "Efecto de ondas concentricas en la ciudad. El impacto de los tuneles de la M-30 medido en el mercado inmobiliario limftrofe". Revista de Obras Publicas 163 (3576): 63-68.

Perez-Prada, F., y A. Monzon. 2014. "How lowering speed limits in an urban highway affects traffic perfirmance and emissions: the caso of Madrid M-30 ring-road". En XI Congreso de Ingeniería del Transporte, CIT 2014, 1-16. Santander, Spain.

Perez-Prada, F., y Monzon, A. 2017. "Ex-post environmental and traffic assessment of a speed reduction strategy in Madrid's inner ring-road". Journal of Transport Geography 58: 256-68. https://doi.org/10.1016/j.jtrangeo.2016.12.018.

Perini, K., y P. Sabbion. 2016. "Madrid Río, Spain - Strategies and Techniques". En Urban Sustainability and River Restoration, 117-26. Chichester, UK: John Wiley \& Sons Ltd. https://doi.org/10.1002/9781119245025.ch11.

Pons, O., y G. Wadel. 2011. "Environmental impacts of prefabricated school buildings in Catalonia". $\quad$ Habitat International $35 \quad$ (4): https://doi.org/10.1016/j.habitatint.2011.03.005.

Pujadas, P., F. Pardo-Bosch, A. Aguado-Renter, y A. Aguado. 2017. "MIVES multi-criteria approach for the evaluation, prioritization, and selection of public investment projects. A case study in the city of Barcelona". Land Use Policy 64: 29-37. https://doi.org/10.1016/j.landusepol.2017.02.014.

Rey Llorente, I., E. Alarcón Álvarez, y I. Espinosa Antelo. 2009. "Ventilación en caso de incendio y gestión medioambiental en los túneles de Calle 30". En Ingeo Túneles, editado por Carlos López Jimeno.

Saaty, T. L. 2012. "How to make a decision". International Series in Operations Research and Management Science 175: 1-21. https://doi.org/10.1007/978-1-4614-3597-6_1.

Sánchez, M., N. López-Mosquera, F. Lera-López, y J. Faulin. 2018. "An Extended Planned Behavior Model to Explain the Willingness to Pay to Reduce Noise Pollution in Road Transportation”. Journal of Cleaner Production 177: 144-54. https://doi.org/10.1016/j.jclepro.2017.12.210.

Silva F., A. Da. 2018. "El río Manzanares en su paso por los tejidos urbanos de Madrid: el juego de posibilidades entre una biofilia hídrica cotidiana y la recuperación ecológica riparia". Territorios en formación, núm. 14: 20-39. https://doi.org/10.20868/tf.2019.14.3890.

Stojčić, M., E. Kazimieras Zavadskas, D. Pamučar, Ž. Stević, y A. Mardani. 2019. "Application of MCDM methods in sustainability engineering: A literature review 2008-2018”. Symmetry 11 (3). https://doi.org/10.3390/sym11030350.

Tatari, O., y D. Kurmapu. 2011. "Sustainability assessment of highways: A Malmquist index of U.S. states". Proceedings of the 2011 IEEE International Symposium on Sustainable Systems and Technology, ISSST 2011. https://doi.org/10.1109/ISSST.2011.5936849.

Viñolas Prat, B., F. Cortés, A. Marques, A. Josa Garcia-Tornel, y A. Aguado de Cea. 2009. "Mives: Modelo Integrado De Valor Para Evaluaciones De Sostenibilidad - ICSMM 2009”. En II Congrés Internacional de Mesura I Modelització de La Sostenibilitat, 1-24. Barcelona, Spain: Centro Internacional de Métodos Numéricos en Ingeniería (CIMNE). https://upcommons.upc.edu/handle/2117/9704\%0Ahttps://www.etcg.upc.edu/prj/mives/herr amienta-mives.

Zhou, F. P., F. D. Lydon, y B. I.G. Barr. 1995. "Effect of coarse aggregate on elastic modulus and compressive strength of high performance concrete". Cement and Concrete Research 25 (1): 177-86. https://doi.org/10.1016/0008-8846(94)00125-I. 